\title{
DESSORÇÃO E CALOR ISOSTÉRICO DE AMÊNDOAS DE BARU
}

\section{DESORPTION AND ISOSTERIC HEAT OF BARU ALMONDS}

\author{
Guilherme de Figueiredo Furtado ${ }^{1}$, Fabrício Schwanz da Silva², Alexandre Gonçalves Porto ${ }^{3}$, Philipe dos \\ Santos ${ }^{4}$. \\ ${ }^{1,4}$ Universidade Estadual de Campinas - UNICAMP - Campinas - Brasil \\ ${ }^{2,3}$ Universidade do Estado de Mato Grosso - UNEMAT - Barra do Bugres - Brasil \\ furtado.gf@gmail.com
}

\begin{abstract}
Resumo
O presente trabalho teve como objetivo determinar as isotermas de dessorção e o calor isostérico de amêndoas de baru sob condições de temperatura e umidade relativa do ar variando de 20, 30 , 40 e $50{ }^{\circ} \mathrm{C}$ e 4,3 a 82,9\% respectivamente. Foram ajustados cinco modelos matemáticos aos dados experimentais com a finalidade de se obter os parâmetros desses modelos e escolher aquele que melhor representasse as isotermas e para o cálculo do calor isostérico. A escolha do melhor ajuste deu-se em função do coeficiente de determinação $\left(R^{2}\right)$ e do desvio médio relativo $(P)$. De acordo com os resultados o modelo de Halsey foi o que melhor se ajustou às isotermas de dessorção, podendo ser empregado para o cálculo da umidade de equilíbrio higroscópico de amêndoas de baru e o calor isostérico variou de 443,33 a $631,91 \mathrm{~kJ}^{\mathrm{kg}} \mathrm{kg}^{-1}$.
\end{abstract}

Palavras-chave: isotermas, modelos matemáticos, atividade de água.

\section{Introdução}

O baru ou barueiro (Dipteryx alata Vogel, Leguminosae Faboideae), também conhecido como cumbaru, é uma espécie leguminosa arbórea que ocorre no Cerrado do Brasil, sendo valorizada por suas diversas utilizações. Possui um fruto de casca fina onde se esconde uma amêndoa dura e comestível, rica em cálcio, fósforo e manganês. Contém $45 \%$ de óleos, o valor protéico e o gosto se assemelham ao amendoim. É consumida preferencialmente torrada e, neste caso, substitui com equivalência a castanha-de-caju, em receitas alimentícias (FERREIRA, 1980). Embora tenham bom potencial econômico, fruto e amêndoas ainda não são comercializados em larga escala. 
É imprescindível, para garantir a qualidade e sua conservação, que os produtos de origem vegetal sejam transportados e armazenados em locais secos e ventilados e, sobretudo, com baixos teores de umidade; do contrário, o desenvolvimento de microrganismos pode causar fermentações indesejáveis e contaminações por toxinas, que inviabilizam a utilização do produto para consumo humano e animal (SAUER, 1992).

A disponibilidade de água em materiais biológicos, tais como grãos e frutos, é melhor indicada pela atividade de água (Aw), ou pela umidade de equilíbrio com a temperatura e umidade relativa do ar ambiente. A atividade de água e a umidade relativa, quando atingido o equilíbrio dinâmico, são numericamente iguais (HALL, 1980; BROOKER et al., 1992) e sua diminuição com a desidratação do produto contribui para a conservação e uso prolongado do material, uma vez que a retirada de água reduz a sua atividade nos produtos biológicos, retardando o crescimento de microrganismos e impedindo a efetivação de reações bioquímicas que aceleram o processo degenerativo evitando, consequientemente, a rápida perda de qualidade do produto.

Os produtos agrícolas possuem a propriedade de realizar trocas de água sob a forma de vapor, com o ambiente que os envolve. Essas trocas podem ocorrer através do ganho ou da perda de água, fenômenos conhecidos, respectivamente, por adsorção e dessorção, de acordo com as características higroscópicas recíprocas do produto e do ar. A afinidade existente entre a água e os outros componentes (gordura, amido, açúcar, proteínas etc.) de um produto, define sua higroscopicidade (BROOKER et al., 1992).

O estudo das curvas de sorção que relacionam a atividade de água e o teor de umidade de equilíbrio a uma dada temperatura, fornece informações fundamentais para adequação das diversas etapas que sucedem a colheita dos produtos agrícolas (AFONSO JÚNIOR, 2001).

Tendo em vista a importância do conhecimento do comportamento higroscópico de produtos agrícolas para avaliar as variáveis de processamento e definir condições de umidade para manutenção de suas qualidades durante o armazenamento, vários pesquisadores vêm empregando grandes esforços para obtenção de equações e modelos matemáticos que melhor representem este fenômeno (AGUERRE et al., 1989; CORRÊA et al., 1998; CHEN; JAYAS, 1998).

O conhecimento do calor isostérico é de grande importância nos projetos de equipamentos de secagem, devido ao fato que o calor de vaporização da água sorvida é mais alto que o calor de vaporização da água pura, quando o alimento é desidratado a baixos níveis de umidade (KING, 1968). Wang e Brennan (1991) descrevem o calor isostérico como um indicador das forças atrativas intermoleculares entre os sítios de sorção e o vapor de água.

O respectivo trabalho teve como objetivo determinar as isotermas de equilíbrio e o calor isostérico de amêndoas de baru, através do estudo da atividade de água e das curvas de dessorção 
nas temperaturas de $20,30,40$ e $50^{\circ} \mathrm{C}$, a uma faixa de umidade relativa de 4,3 a $82,9 \%$, utilizandose do método gravimétrico estático.

\section{Material e Métodos}

Este estudo foi desenvolvido no Laboratório de Engenharia e Processamento Agroindustrial pertencente ao centro Tecnológico do Mato Grosso, localizado no Campus Universitário Dep. Estadual Renê Barbour da Universidade do Estado de Mato Grosso, na cidade de Barra do Bugres, Mato Grosso.

Foram utilizados como matéria prima frutos de baru que foram coletados na região e tiveram suas amêndoas extraídas de acordo com metodologia descrita por (KOYUNCU et al., 2000).

O delineamento experimental foi inteiramente casualizado, num esquema fatorial $4 \times 10$, sendo quatro níveis de temperatura $\left(20,30,40\right.$ e $\left.50{ }^{\circ} \mathrm{C}\right)$ e dez níveis de concentrações de solução de ácido sulfúrico $(25,30,35,40,45,50,55,60,65$ e 70\%) em um total de três repetições. A Tabela 1 apresenta a atividade de água em função das temperaturas e concentrações ácidas, utilizadas no respectivo experimento.

Tabela 1 - Valores de atividade de água para as concentrações de $\mathrm{H}_{2} \mathrm{SO}_{4}$ em diferentes temperaturas.

\begin{tabular}{ccccc}
\hline Conc. $\mathrm{H}_{2} \mathrm{SO}_{4}(\%)$ & $20{ }^{\circ} \mathrm{C}$ & $30{ }^{\circ} \mathrm{C}$ & $40{ }^{\circ} \mathrm{C}$ & $50{ }^{\circ} \mathrm{C}$ \\
\hline 25 & 0,816 & 0,817 & 0,824 & 0,829 \\
30 & 0,749 & 0,747 & 0,753 & 0,77 \\
35 & 0,665 & 0,666 & 0,674 & 0,681 \\
40 & 0,568 & 0,565 & 0,574 & 0,582 \\
45 & 0,458 & 0,461 & 0,47 & 0,483 \\
50 & 0,355 & 0,355 & 0,366 & 0,38 \\
55 & 0,258 & 0,26 & 0,267 & 0,28 \\
60 & 0,167 & 0,17 & 0,178 & 0,189 \\
65 & 0,093 & 0,097 & 0,102 & 0,11 \\
70 & 0,043 & 0,045 & 0,049 & 0,055 \\
\hline
\end{tabular}

Fonte: Perry e Chilton, 1983.

O método gravimétrico estático foi utilizado de acordo com metodologia descrita por Porto (2001) onde as amostras foram inseridas em pequenos recipientes plásticos, dentro de recipientes de vidro com as soluções ácidas. O aparato permaneceu em estufa incubadora com temperatura controlada até atingir massa constante, logo após foi determinado o teor de umidade das amostras pelo método da estufa $105^{\circ} \mathrm{C}$ por 24 horas. 
Posteriormente, foram ajustados modelos matemáticos utilizados por Corrêa et al. (1998) e Gouveia, et al. (2004), apresentados na Tabela 2, aos dados experimentais por análise de regressão utilizando-se um programa computacional de análise estatística.

Tabela 2 - Modelos matemáticos de sorção.

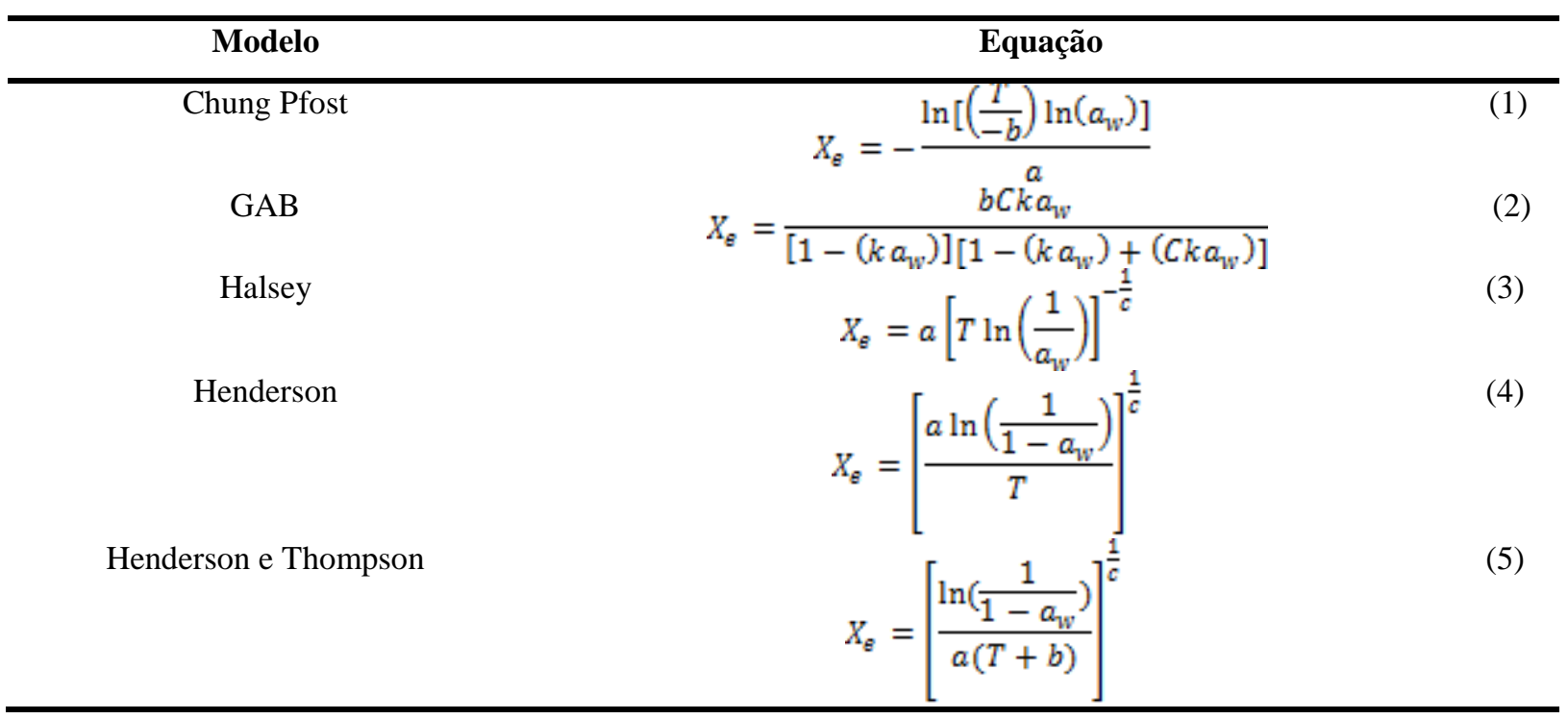

Onde:

$\mathrm{T}=$ temperatura ${ }^{\circ} \mathrm{C} \quad \mathrm{X}_{\mathrm{e}}=$ umidade de equilíbrio, b.s.

$\mathrm{a}_{\mathrm{w}}=$ atividade de água $\quad c=c * e^{\left(\frac{d}{T}\right)}$

$k=a * e^{\left(\frac{e}{T}\right)} \quad \mathrm{a}, \mathrm{b}, \mathrm{c}, \mathrm{d}, \mathrm{e}=$ parâmetro de ajuste

Os dados experimentais foram comparados com os valores calculados pelos modelos matemáticos, analisando-se os coeficientes de determinação $\left(\mathrm{R}^{2}\right)$ e o erro médio relativo (P) (Eq. 6), para analisar qual modelo se ajustou melhor aos resultados obtidos. Para o cálculo do calor isostérico $\left(\mathrm{Q}_{\mathrm{st}}\right)$ fez-se uma regressão linear dos dados do logaritmo neperiano da atividade de água com o recíproco da temperatura a diferentes umidades de equilíbrio, e se aplicou a equação de Clausius-Clapeyron (Eq. 7); em seguida, testou-se o modelo exponencial de Sopade e Ajisegiri (1994) (Eq. 8) que prediz o comportamento do calor isostérico em função do conteúdo de umidade de equilíbrio.

$P=\frac{100}{n} \sum\left(\frac{Y-Y^{n}}{Y}\right)$ 
$Q_{s t}=-R\left[\frac{d\left(\ln \left(a_{w}\right)\right.}{d\left(\frac{1}{T}\right)}\right]$

$Q_{s t}=A e^{\left(B X_{e}\right)}$

Onde:

$\mathrm{n}=$ número de observações experimentais

$\mathrm{Y}=$ valor observado experimentalmente

$\mathrm{Y}^{\wedge}=$ valor calculado pelo modelo

$\mathrm{Q}_{\mathrm{st}}=$ calor isostérico, $\mathrm{kJ} \cdot \mathrm{kg}^{-1}$

$\mathrm{A}$ e $\mathrm{B}=$ parâmetros de ajuste , admensionais

$\mathrm{R}=$ constante universal dos gases

\section{Resultados e Discussão}

Na Tabela 3, encontram-se os valores obtidos experimentalmente para a dessorção, representando a umidade de equilíbrio das amêndoas de baru, submetidas às condições ambientais em soluções ácidas, em função da atividade de água, variando de 4,3\% a 82,9\% e da temperatura, $20,30,40$ e $50^{\circ} \mathrm{C}$.

Tabela 3 - Umidade de equilíbrio $(\mathrm{Xe})$ a diferentes atividades de água $\left(\mathrm{a}_{\mathrm{w}}\right)$ temperaturas.

\begin{tabular}{|c|c|c|c|c|c|c|c|}
\hline \multicolumn{8}{|c|}{ Temperatura $\left({ }^{\circ} \mathrm{C}\right)$} \\
\hline \multicolumn{2}{|c|}{20} & \multicolumn{2}{|c|}{30} & \multicolumn{2}{|c|}{40} & \multicolumn{2}{|c|}{50} \\
\hline$\overline{a_{w}}$ & $\overline{X_{e}}$ & $\overline{a_{w}}$ & $\overline{X_{e}}$ & $\overline{a_{w}}$ & $\mathrm{X}_{\mathrm{e}}$ & $\overline{a_{w}}$ & $\overline{X_{e}}$ \\
\hline 0,816 & 0,12 & 0,817 & 0,14 & 0,824 & 0,16 & 0,829 & 0,18 \\
\hline 0,749 & 0,10 & 0,747 & 0,12 & 0,753 & 0,12 & 0,770 & 0,14 \\
\hline 0,665 & 0,09 & 0,666 & 0,10 & 0,674 & 0,10 & 0,681 & 0,11 \\
\hline 0,568 & 0,08 & 0,565 & 0,09 & 0,574 & 0,08 & 0,582 & 0,08 \\
\hline 0,458 & 0,07 & 0,461 & 0,08 & 0,470 & 0,06 & 0,483 & 0,07 \\
\hline 0,355 & 0,07 & 0,355 & 0,07 & 0,366 & 0,05 & 0,380 & 0,06 \\
\hline 0,258 & 0,06 & 0,260 & 0,06 & 0,267 & 0,04 & 0,280 & 0,05 \\
\hline 0,167 & 0,06 & 0,170 & 0,05 & 0,178 & 0,04 & 0,189 & 0,04 \\
\hline 0,093 & 0,05 & 0,097 & 0,05 & 0,102 & 0,03 & 0,110 & 0,03 \\
\hline 0,043 & 0,05 & 0,045 & 0,04 & 0,049 & 0,03 & 0,055 & 0,02 \\
\hline
\end{tabular}

Os modelos matemáticos e seus parâmetros apresentados anteriormente foram ajustados a esses dados para que posteriormente fosse escolhido o modelo que melhor representasse tal fenômeno.

$\mathrm{Na}$ Tabela 4, encontram-se os parâmetros dos modelos testados e ajustados para as amêndoas de baru, em função das umidades relativas e temperaturas estudadas, bem como os coeficientes de determinação $\left(\mathrm{R}^{2}\right)$ e o erro médio relativo $(\mathrm{P})$. 
Tabela 4 - Parâmetros de ajuste das isotermas de dessorção em amêndoas de baru submetidas à soluções ácidas para os diferentes modelos matemáticos.

\begin{tabular}{|c|c|c|c|c|c|c|c|c|}
\hline MODELO & $\begin{array}{l}\text { Temp. } \\
\left({ }^{\circ} \mathrm{C}\right)\end{array}$ & $\mathrm{a}$ & $\mathrm{b}$ & $\mathrm{c}$ & $\mathrm{D}$ & E & $\mathrm{R}^{2}$ & $\mathrm{P}$ \\
\hline \multirow{4}{*}{ (1) } & 20 & 53,866 & 366,520 & & & & 0,947 & 7,419 \\
\hline & 30 & 43,210 & 260,355 & & & & 0,967 & 9,286 \\
\hline & 40 & 43,467 & 179,984 & & & & 0,903 & 23,913 \\
\hline & 50 & 32,343 & 156,121 & & & & 0,970 & 20,588 \\
\hline \multirow{4}{*}{ (2) } & 20 & 1,986 & 0,036 & 2493,259 & 222,827 & $-20,095$ & 0,98 & 4,558 \\
\hline & 30 & 0,854 & 0,031 & 243,38 & 16,481 & $-1,207$ & 0,997 & 32,515 \\
\hline & 40 & 0,407 & 0,304 & 0,415 & 3,62 & $-1,47$ & 0,876 & 32,515 \\
\hline & 50 & 0,43 & 0,309 & 0,425 & 3,873 & $-1,512$ & 0,974 & 22,354 \\
\hline \multirow{4}{*}{ (3) } & 20 & 0,143 & & 2,929 & & & 0,977 & 4,991 \\
\hline & 30 & 0,210 & & 2,255 & & & 0,994 & 4,092 \\
\hline & 40 & 0,366 & & 1,476 & & & 0,988 & 9,612 \\
\hline & 50 & 0,515 & & 1,366 & & & 0,995 & 7,978 \\
\hline \multirow{4}{*}{ (4) } & 20 & 0,165 & & 3,487 & & & 0,905 & 10,619 \\
\hline & 30 & 0,276 & & 2,463 & & & 0,947 & 12,621 \\
\hline & 40 & 0,786 & & 1,364 & & & 0,913 & 25,903 \\
\hline & 50 & 1,36 & & 1,241 & & & 0,984 & 15,384 \\
\hline \multirow{4}{*}{ (5) } & 20 & 114,253 & 91,840 & 3,546 & & & 0,905 & 10,384 \\
\hline & 30 & 24,524 & 1,219 & 2,487 & & & 0,947 & 12,491 \\
\hline & 40 & 1,371 & 0,698 & 1,365 & & & 0,913 & 25,892 \\
\hline & 50 & 0,600 & 0,261 & 1,195 & & & 0,985 & 16,629 \\
\hline
\end{tabular}

Observa-se na Tabela 4 que o modelo de Halsey descreveu com maior precisão as isotermas de dessorção para as amêndoas de baru, pois apresentou os menores coeficientes de determinação e erro médio relativo, e segundo Lomauro et al. (1985), o melhor modelo é aquele que possui os maiores valores de $\mathrm{R}^{2}$ e erro médio relativo $(\mathrm{P})$ menor que $10 \%$. Na Figura 1 são apresentados os gráficos de correspondência entre os teores de água observados e os estimados pelos modelos analisados. Esses gráficos permitem confirmar o excelente ajuste do modelo de Halsey aos dados observados. 
Figura 1 - Valores de correspondência entre valores preditos e observados pelos modelos analisados para as isotermas de dessorção de amêndoas de baru.

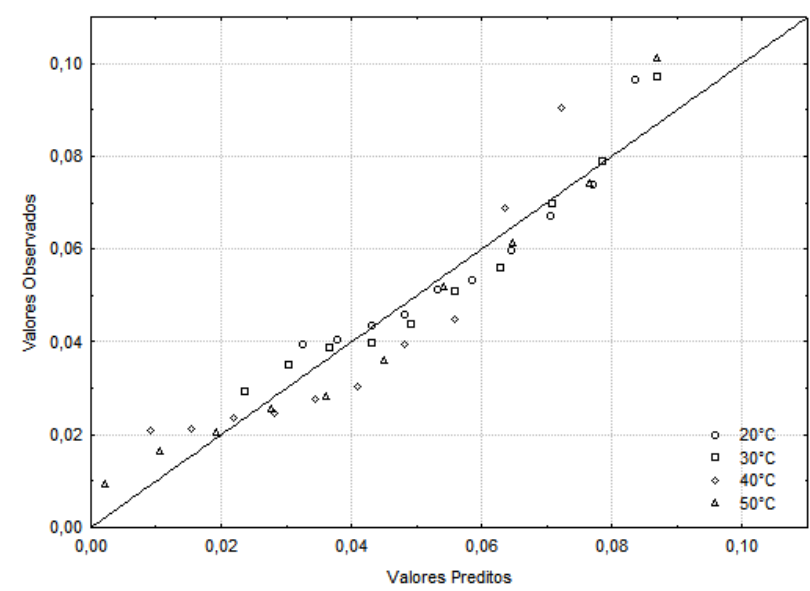

Chung Pfost

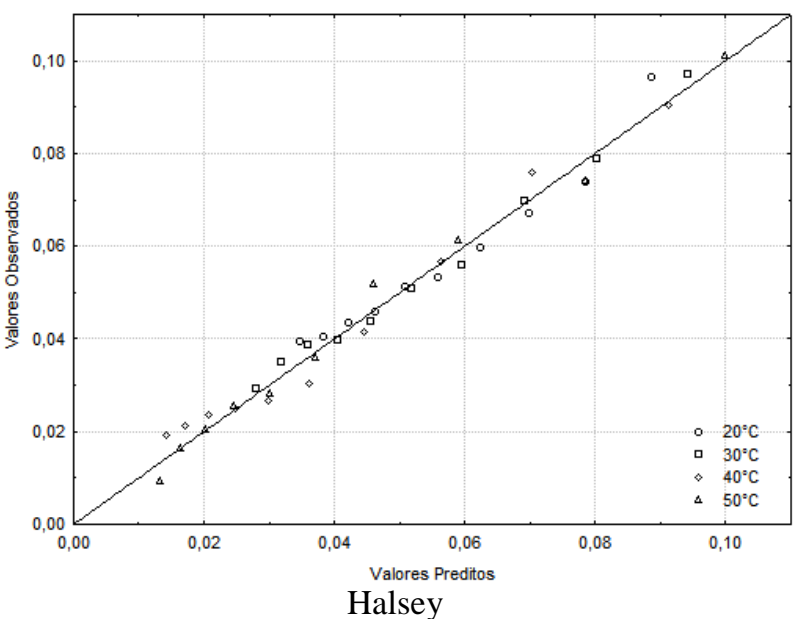

Halsey

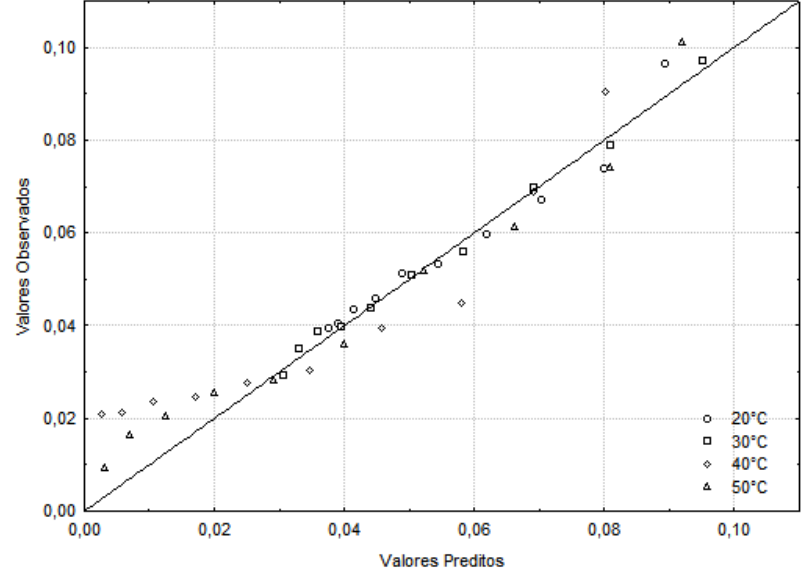

GAB

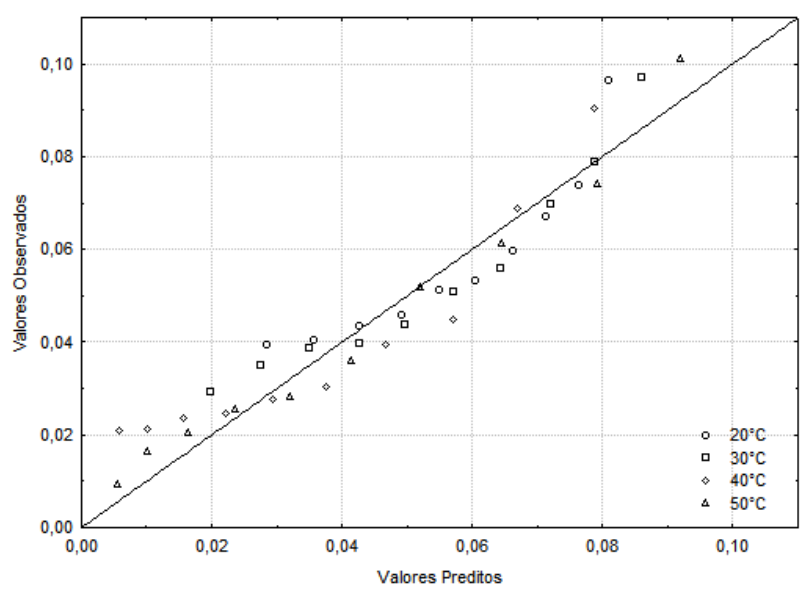

Henderson

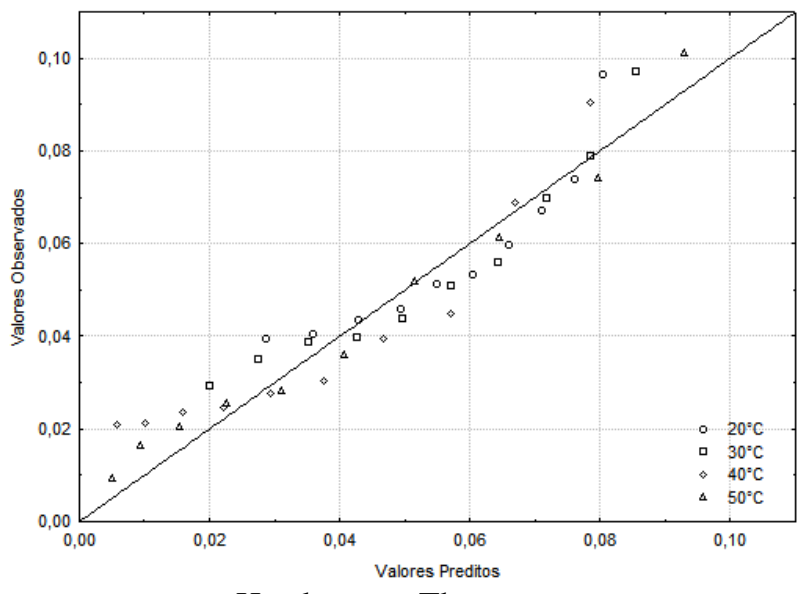

Henderson e Thompson

Os resultados obtidos a partir do modelo de Halsey estão representados graficamente na Figura 2 demonstrando a influência da temperatura e da atividade de água sobre a umidade de equilíbrio do alimento. 
Figura 2. Isotermas de dessorção de amêndoas de baru nas temperaturas de $20,30,40$ e $50^{\circ} \mathrm{C}$, segundo o modelo Halsey.

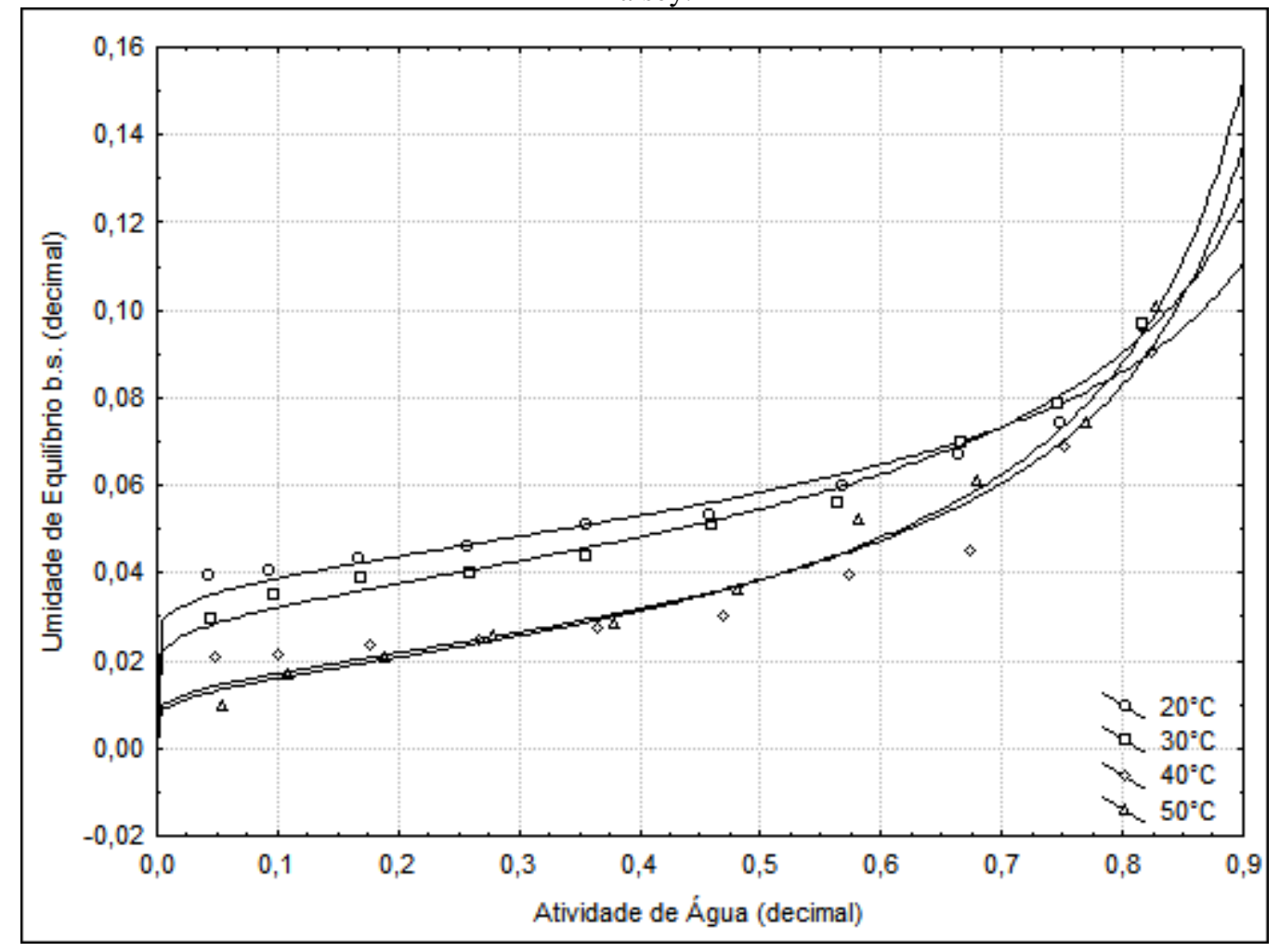

O comportamento da umidade de equilíbrio respondeu de forma diretamente proporcional ao aumento das atividades de água fixadas pelo método gravimétrico estático utilizando-se soluções ácidas, para todas as temperaturas.

Pena et al. (2009) estudando as isotermas de adsorção e dessorção do resíduo da industrialização do suco de maracujá submetido a secagem em camada delgada concluíram que com base nos valores de $\mathrm{R}^{2}$ e $\mathrm{P}(\mathrm{P}<10 \%)$, constatou-se que a equação de Halsey foi uma das equações que apresentou os melhores ajustes, sendo indicadas para a predição das isotermas de adsorção e dessorção do produto estudado.

Lehn et al. (2004), estudando as isotermas de equilíbrio com ácidos e curvas de secagem de arroz em casca, dentre quatro modelos estudados, concluíram que a equação de Halsey apresentou o melhor ajuste aos dados de umidade de equilíbrio, nas temperaturas de $10^{\circ} \mathrm{C}, 15^{\circ} \mathrm{C}$ e $20^{\circ} \mathrm{C}$, além de apresentar comportamento sigmoidal, afirmando a influência da temperatura e da atividade de água às amostras, semelhantemente ao observado no respectivo trabalho.

As curvas isostéricas de sorção (ln $\left.\left[\mathrm{a}_{\mathrm{w}}\right]\right)$ versus $1 / \mathrm{T}$ em função da umidade de equilíbrio das amostras apresentadas na Figura 3, foram obtidas baseadas nos dados das curvas de equilíbrio higroscópico ajustadas pelo modelo de Halsey. 
Figura 3. Curvas de $\ln \left(\mathrm{a}_{\mathrm{w}}\right)$ versus $1 / \mathrm{T}$, baseadas na equação de Clausius-Clapeyron.

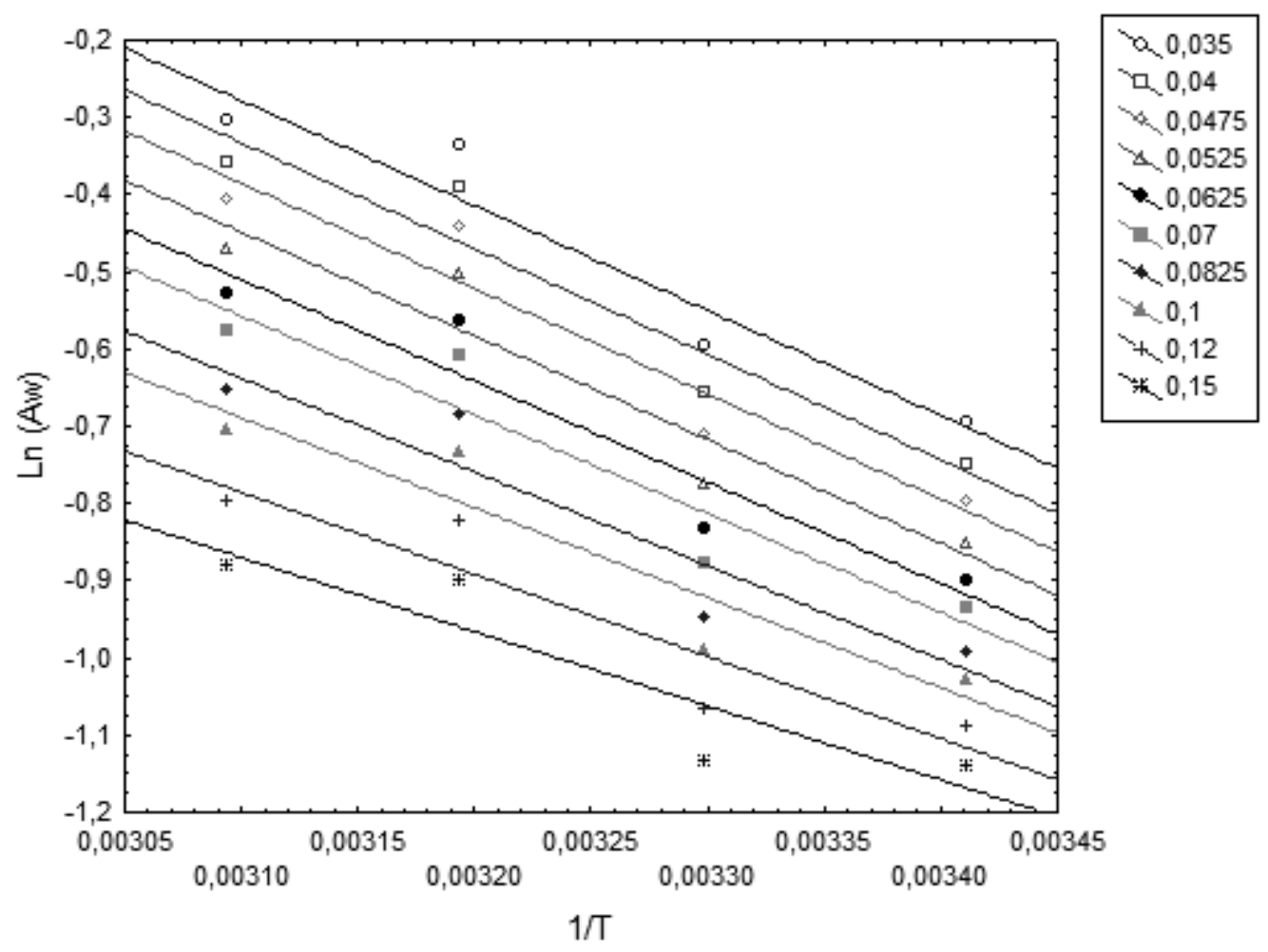

Na Tabela 5 encontram-se os valores do calor isostérico para cada conteúdo de umidade. Estes valores correspondem aos coeficientes de inclinação das retas da Figura 3, multiplicados pela constante universal dos gases $\left(\mathrm{R}=0,4618 \mathrm{~kJ} \cdot \mathrm{kg}^{-1} \mathrm{~K}^{-1}\right)$ conforme propõe a equação de ClausiusClapeyron. Zhang et al. (1996), estudando isotermas de sorção de umidade de alguns vegetais nas temperaturas de 30 a $60{ }^{\circ} \mathrm{C}$, concluíram que o calor isostérico de dessorção pode ser utilizado para estimar a energia requerida no processo de desidratação de produtos agrícolas.

Tabela 5. Valores do calor de sorção das amêndoas de baru.

\begin{tabular}{cc}
\hline$X_{\mathrm{e}}$ (b.s.) & $\mathrm{Q}_{\mathrm{ST}}\left(\mathrm{kJkg}^{-1}\right)$ \\
\hline 0,0350 & 627,61 \\
0,0400 & 631,91 \\
0,0475 & 629,61 \\
0,0525 & 620,16 \\
0,0625 & 605,23 \\
0,0700 & 590,65 \\
0,0825 & 560,28 \\
0,1000 & 537,95 \\
0,1200 & 490,03 \\
0,1500 & 443,33 \\
\hline
\end{tabular}

De acordo com a Tabela 5, observa-se que o calor isostérico diminui com o aumento do conteúdo de água do produto, indicando que a redução do teor de água aumenta a energia necessária para a remoção de água. Wang e Brennan (1991) justificam que em produtos com menor umidade as forças de ligação entre a água e a superfície da substância adsorvente são maiores. Resende et al. 
(2006) e Moraes et al. (2007) encontraram resultado semelhante com o estudo de isotermas e calor isostérico de sorção do feijão e quitina respectivamente.

Para o ajuste da curva do calor de isostérico $\left(\mathrm{Q}_{\mathrm{st}}\right)$ em função da umidade de equilíbrio $(\mathrm{X})$, utilizou-se o modelo matemático exponencial de Sopade e Ajisegiri (1994). O gráfico do calor isostérico em função da umidade encontra-se na Figura 4.

Figura 4. Calor isostérico em função do conteúdo de umidade das amêndoas de baru, de acordo com a equação de Sopade \& Ajisegiri (1994).

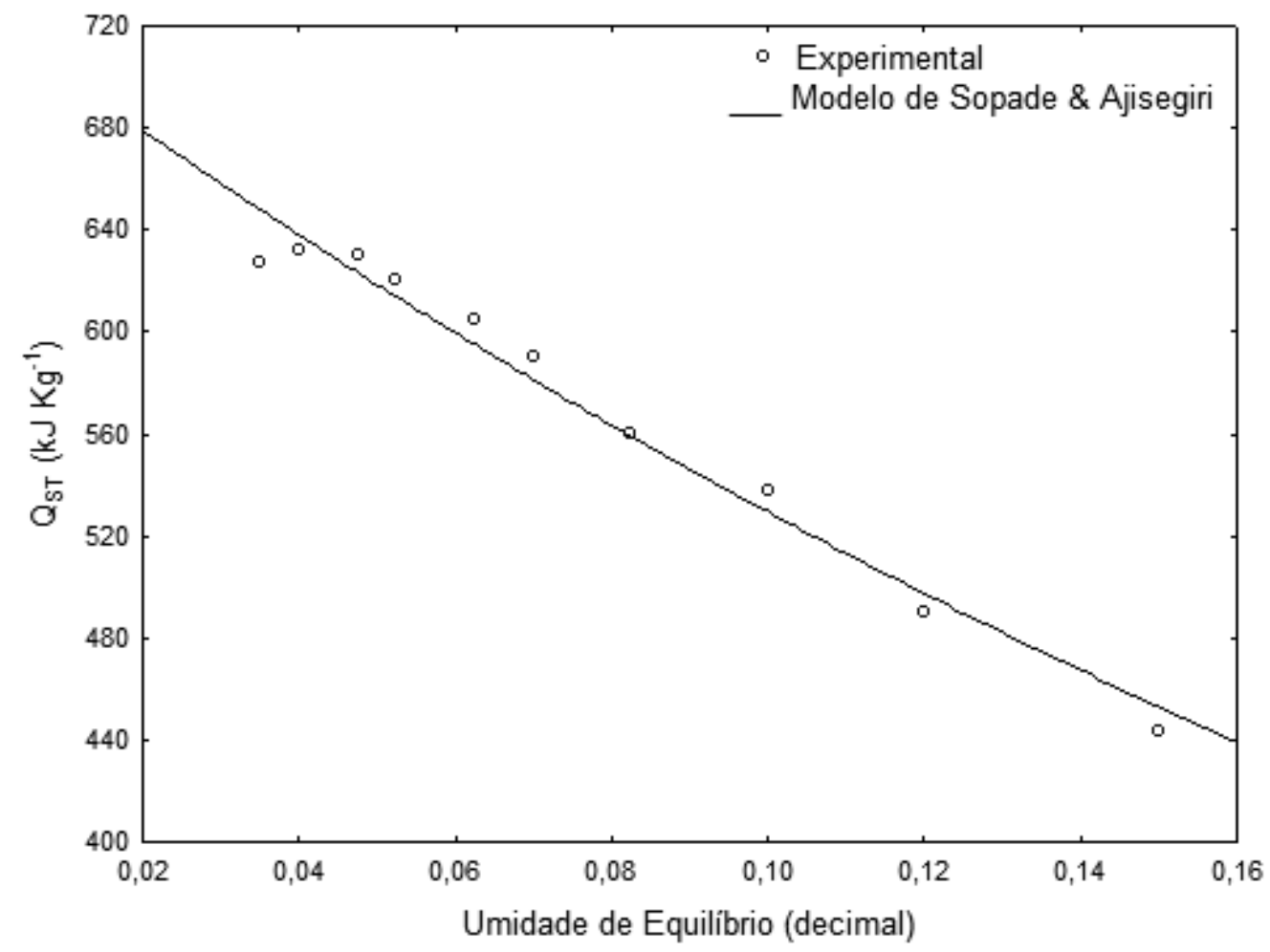

Os parâmetros calculados para este modelo, foram $\mathrm{A}=722,74$ e $\mathrm{B}=-3,12$; e o coeficiente de determinação $\left(\mathrm{R}^{2}\right)$ e o erro médio relativo $(\mathrm{P})$ foram de 0,987 e 0,015, respectivamente. Em virtude dos valores de $\mathrm{R}^{2}$ e $\mathrm{P}$ encontrados, pode-se dizer que o modelo faz uma estimativa adequada dos valores do calor de sorção de amêndoas de baru.

\section{Conclusão}

Os valores de umidade de equilíbrio diminuíram com o aumento da temperatura e com a diminuição das atividades de água fixadas pelo método gravimétrico estático utilizando-se soluções ácidas.

O modelo matemático de Halsey foi o que melhor se ajustou às isotermas de dessorção de amêndoas de baru. 
O calor isostérico variou de 443,33 a 631,91 kJ.kg-1 e diminuiu com o aumento da umidade de equilíbrio.

\begin{abstract}
This study aimed to determine the desorption isotherms and isosteric heat of Baru almonds under conditions of temperature and relative humidity ranging from 20,30, 40 and $50{ }^{\circ} \mathrm{C}$ and 4,3 to $82,9 \%$ respectively. Five mathematical models were fitted to experimental data for the purpose of obtaining the model parameters and choose the one that best represented the isotherms and to calculate the isosteric heat. Choosing the best fit was based on the coefficient of determination $\left(R^{2}\right)$ and the relative mean deviation $(P)$. According to the results Halsey model was the best fitted to the desorption isotherms, could be used to calculate the hygroscopic equilibrium moisture content of

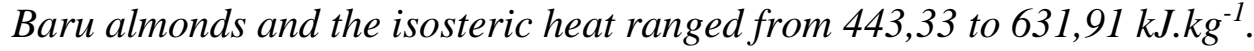

Key-words: isotherms, mathematical models, water activity.

\title{
Referências
}

AFONSO JÚNIOR, P. C. Influência do método de preparo e condições de secagem sobre a qualidade do café durante o período de armazenagem. 2001, 351p., Tese Doutorado, UFV, Viçosa.

AGUERRE, R. J.; SUAREZ, C.; VIOLLAZ, P. E. New BET type multi-layer sorption isotherms - Part II: Modeling water sorption in foods. Lebensmittel-wissenschaft und technologie , London, v. 22, n. 4, p. 192-195, 1989.

BROOKER, D. B.; BAKKER-ARKEMA, F. W.; HALL, C. W.; Drying and storage of grains and oilseeds. Westport, The AVI Publishing Company, New York, 1992.

CHEN, C.; JAYAS, D. S. Evaluation of the GAB equation for the isotherms of agricultural products. Transactions of ASAE, St. Joseph, v. 41, n. 6, p. 1755-1760, 1998

CORRÊA, P. C.; MARTINS, J. H.; CHRIST, D.; MANTOVANI, B. H. M. Curvas de dessorção e calor latente de vaporização para as sementes de milho pipoca (Zea mays). Revista Brasileira de Engenharia Agrícola e Ambiental, Campina Grande, v. 2, n. 1, p. 75-79, 1998.

FERREIRA, M. B. Frutos comestíveis nativos do cerrado em Minas Gerais. Informe Agropecuário, Belo Horizonte, v. 6, n. 61, p. 9-18, 1980.

GOUVEIA, J. P. G., NAScimento, J., AlmeidA, F. A. C., SIlVA, M. M., FARIAS, A. S., SILVA, F. L. H.. Modelos matemáticos para ajuste das isotermas de dessorção da polpa de banana da variedade prata. Revista de Engenharia Agrícola, Jaboticabal, v. 24, n. 3, p. 799-806, 2004.

HALL, C. W. Drying and storage of agricultural crops. Westport: The AVI Publishing Company, 1980.

KING, C. J. Rate of moisture sorption and desorption in porous, dried foodstuffs. Food Technology, v. 22, p. 509-514, 1968.

KOYUNCU, M. A.; EKINCI, K.; SAVRAN, E. Cracking Characteristics of Walnut. Biosystems Enginnering. v. 87, n. 3, p. 305-311, 2004. http://dx.doi.org/10.1016/j.biosystemseng.2003.11.001

LEHN, D. N., PINTO, L. A. de A., Isotermas de equilíbrio e curvas de secagem para arroz em casca em silos de armazenagem. Engenharia na Agricultura, Viçosa, MG, v. 12, n. 3, p. 177-191, 2004.

LOMAURO, C. J.; BAKSHI, A. S.; LABUZA, T. P. Moisture transfer properties of dry and semimoist foods. Journal of Food Science, v. 50, n. 2, p. 397-400, 1985. http://dx.doi.org/10.1111/j.1365-2621.1985.tb13411.x

MORAES, M. A.; ROSA, G. S.; PINTO, L. A. A. Isotermas de Equilíbrio para Quitina: Determinação do Calor de Dessorção. Brazilian Journal of Food Technology, v. 10, n. 3, p. 212-219, 2007. 
PENA, R. S. ; MENDONÇA, N. B. Secagem em camada delgada da fibra residual do maracujá. Boletim do Centro de Pesquisa e Processamento de Alimentos, v. 27, p. 257-270, 2009.

PERRY, R. H.; CHILTON, C. H., Chemical Engineers' Handbook, McGraw-Hill Co., 5 ed., Sec. 20-4. 1983.

PORTO, P. S. S. Cinética da secagem de cebola em camada delgada. 2001, 123p., Dissertação de Mestrado. FURG, Rio Grande, RS.

RESENDE, O.; CORREA, P. C.; GONELI, A. L. D.; RIBEIRO, D. M. Isotermas e calor isostérico de sorção do feijão. Ciência e Tecnologia de Alimentos, v. 26, n. 3, p. 626-631, 2006. http://dx.doi.org/10.1590/S010120612006000300022

SAUER, D. B. Storage of cereal grains and their products. St Paul: American Association of Cereal Chemists, 1992. $615 \mathrm{p}$.

SOPADE, P.A.; AJISEGIRI, E.S. Moisture sorption study on nigerian foods: maize and sorghum. Journal of Food Engineering, Lleida, v. 17, n. 1, p. 33-56, 1994. http://dx.doi.org/10.1111/j.1745-4530.1994.tb00327.x

WANG, N.; BRENNAN, J.G. Moisture sorption isotherm characteristics of potato at four temperatures. Journal of Food Engineering, v. 14, p. 269-287, 1991. http://dx.doi.org/10.1016/0260-8774(91)90018-N

ZHANG, X.; LIU, X.; GU, D.; ZHOU, W.; WANG, R.; MARINOS-KOURIS, D. Desorption isotherms of some vegetables. Journal of Science and Food Agriculture, v. 70, p. 303-306, 1996.

Submetido em 30 jul. 2013; Aceito para publicação em 14 abr. 2014. 